\title{
Silicone breast phantoms for elastographic imaging evaluation
}

Amer S. Kashif

Centre for Bioengineering, Dept of Mechanical Engineering, University of Canterbury, Christchurch,

$5 \quad$ New Zealand

Thomas F. Lotz

Centre for Bioengineering, Dept of Mechanical Engineering, University of Canterbury, Christchurch, New Zealand

Matthew D. McGarry

10 Thayer School of Engineering, Dartmouth College, Hanover NH, USA

Adam J. Pattison

Thayer School of Engineering, Dartmouth College, Hanover NH, USA

James G. Chase $\mathrm{a}^{\mathrm{a})}$

Centre for Bioengineering, Dept of Mechanical Engineering, University of Canterbury, Christchurch, $15 \quad$ New Zealand

(Received 
Purpose: Breast cancer is a major public health issue for women, and early detection significantly increases survival rate. Currently, there is increased research interest in elastographic soft-tissue imaging techniques based on the correlation between pathology and mechanical stiffness. Anthropomorphic breast phantoms are critical for in-vitro validation of emerging elastographic technologies. This research develops heterogeneous breast phantoms for use in testing elastographic imaging modalities.

25 Methods: Mechanical property estimation of eight different elastomers is performed to determine storage moduli (E') and damping ratios (ל) using a dynamic mechanical analyzer (DMA). Dynamic compression testing was carried out isothermally at room temperature over a range of $4-50 \mathrm{~Hz}$. Silicone compositions with physiologically realistic storage modulus were chosen for mimicking skin adipose, cancerous tumors and pectoral muscles and 13 anthropomorphic breast phantoms were constructed for in vitro trials of Digital Image Elasto Tomography (DIET) breast cancer screening system. A simpler fabrication was used to assess the possibility of multiple tumor detection using Magnetic Resonance Elastography (MRE).

Results: Silicone materials with ranges of storage moduli (E') from 2 to $570 \mathrm{kPa}$ and damping ratios (५) from 0.03 to 0.56 were identified. The resulting phantoms were tested in two different elastographic breast cancer diagnostic modalities. A significant contrast was successfully identified between healthy tissues and cancerous tumors both in Magnetic Resonance Elastography (MRE) and Digital Image Elasto-tomography (DIET).

Conclusions: The phantoms presented promise aid to researchers in elastographic imaging modalities for breast cancer detection and provide a foundation for silicone based phantom materials for mimicking soft tissues of other human organs.

Keywords: Elastography, mechanical property estimation, breast phantoms, elastomers, tissue properties 


\section{INTRODUCTION}

\section{I.A. Background}

Pathological changes such as development of different types of carcinomas alter the elastic properties of human tissues resulting in stiffness contrast of up to 5:1 between cancerous tissue and healthy tissues. ${ }^{1-4}$ This contrast has given rise to continued research in the field of elastic parameter estimation of human tissues, ${ }^{1,2,5-18}$ resulting in the development of various elastographic imaging techniques. For breast cancer detection, palpation or clinical breast examination (CBE) remains the most common procedure to date with its inherent limitations of subjectivity and low sensitivity. ${ }^{19,20}$ Other detection modalities include mammography, ultrasound, CT and MRI. Mammography is the only modality currently approved for screening applications, due to its low cost and short duration, in spite of the potential side effects from the radiation.

55 To overcome these limitations, a range of elastographic imaging techniques has been introduced. Mechanical properties of the breast can be estimated in vivo with Ultrasound Elastography (USE) $)^{21-25}$ and Magnetic Resonance Elastography (MRE) ${ }^{26,27}$ The associated cost for MRE is relatively high, rendering it less suitable for large scale screening programs.

Digital Image Elasto Tomography (DIET) is a novel approach of breast screening, which analyses elastic properties of breast tissues and based on the tissue contrast between healthy and cancerous tissues, detects presence of tumor. ${ }^{28-31}$ Low frequency $(5-100 \mathrm{~Hz})$ sinusoidal waves are induced in the breast and the resulting surface oscillations are captured in three dimensions (3D) by an array of digital cameras tracking randomly applied fiducial markers. ${ }^{29,32,33}$ The surface motion is analyzed to detect disturbances in vibration patterns. Areas of higher stiffness within the breast result in a different surface vibration response compared to healthy tissue. This response can be used to determine the location of a potentially cancerous inclusion. With this approach, $10 \mathrm{~mm}$ masses can be localized in silicone phantoms. ${ }^{29}$ Further computation with Finite Element ${ }^{34}$ or Boundary Element inverse problems ${ }^{35}$ can help to more accurately localize the tumor and its size. The key advantages of 
this technology are that it is, portable, low cost, and does not involve breast compression or radiation exposure.

Despite extensive characterization of the elastic properties of structural materials, mechanical properties of many biological materials remain unknown or uncertain, due in part to the technical difficulty of measuring visco-elastic tissues ${ }^{24,36-38}$ (particularly in-vivo), and intersubject variation for a given tissue type. The stiffness of healthy and cancerous breast tissues have been investigated, ${ }^{1,2,39-43}$

75 stiffness contrasts in the range of 5:1 have been reported for carcinomas.

\section{I.B. Breast phantom materials}

Based on the measured elastic properties of human breast tissues, researchers have developed phantoms for validation of elastographic imaging systems. Various materials have been used to mimic human breast tissues and the elastic properties of these materials cover a wide range. Table 1 contains data pertaining to previously reported elastic properties of breast phantoms. It is worth noting that the stiffness parameters are highly dependent on the material used and that the results differ significantly in static and dynamic testing.

Table I. Materials used for mimicking breast tissues and the measured values of Young's modulus by previous investigators

\begin{tabular}{|c|c|c|c|}
\hline & \multirow[t]{2}{*}{ Material used } & \multicolumn{2}{|c|}{ Young's Modulus (kPa) } \\
\hline & & Background & Inclusion \\
\hline Samani \& Plewes ${ }^{40}$ & $\begin{array}{l}\text { Gelatin (Sigma- } \\
\text { Aldrich) }\end{array}$ & 11.74 & 31.30 \\
\hline Xing Liang et $\mathrm{al}^{43}$ & Silicone RTV -615 A & 8.55 & 359.18 \\
\hline Madsen et $\mathrm{al}^{9}$ & Gelatin \& Safflower oil & $16-19$ & $59-72$ \\
\hline Egorov et al ${ }^{39}$ & $\begin{array}{l}\text { Silicone Gel } \\
\text { SEMICOSIL }\end{array}$ & 6 & 75 \\
\hline Peters et al* & $\begin{array}{l}\text { Silicone Gel } \\
\text { A-341C \& LSR-05 }\end{array}$ & $26-33$ & $98-135$ \\
\hline
\end{tabular}

*Peters et al, ${ }^{44}$ measured only the in-phase component of Young's modulus, storage modulus ( $E$ ') 


\section{I.C. Design of anthropomorphic breast phantom}

Breast shaped phantoms of varying tissue properties and tumor location and size can be used to test elastographic imaging modalities of MRE,,$^{17,26,27} \mathrm{USE}^{21-25}$ and DIET. ${ }^{28-34}$ Furthermore, it is desirable to obtain phantoms that are stable over time and easy to fabricate with elastic properties matching human breast tissues. $^{9,39,40,41,44,45}$

90 In this work, a method of creating anthropomorphic breast phantoms of varying elastic and damping properties is presented. Mechanical testing of various silicone compositions was undertaken to compare their elastic properties. Validation was performed on geometrically different example phantoms, using DIET and in MRE research and the phantoms were found to be useful for in vitro trials of both elastographic imaging modalities. It is likely, that the investigated materials may not be usable, for evaluation of USE, especially Acoustic Radiation Force Impulse (ARFI) imaging, due to the low speed of ultrasound propagation and high attenuation coefficients. ${ }^{46}$ 


\section{Materials and Methods}

\section{II.A. Phantom specifications and tissues to model}

During DIET imaging, the patient lies horizontally face down, while the breast is hanging pendant through a hole. The breast is actuated at a range of frequencies $(10-50 \mathrm{~Hz})$ and the breast surface motion is optically imaged through an array of digital cameras. Motion is reconstructed in $3 \mathrm{D}$ to obtain Bode plots and analyze effects of stiffer inclusions on the amplitude of motion, phase disruption and variation in natural frequencies..$^{28,29,44,47-50}$ The phantoms required for in vitro DIET trials, need to mimic an average female breast, both geometrically as well as from stiffness point of view. The size specifications were: $:^{51-53}$

- Approx $110 \mathrm{~mm}$ in diameter at the base (chest wall)

- $65 \mathrm{~mm}$ long in P-A (posterior - anterior) direction

- $\quad$ Taper as illustrated in Figure 1(A).

110 Based on the anatomy of the breast following tissues were required to be modeled:

- Skin: To include a compressive or constraining traction

- Adipose

- Tumor

- Pectoral muscle

\section{II.B. Elastomers used:}

The conventional, two component (base plus curative) silicones, room temperature vulcanizing (RTV) silicones (curable at room temperature) were chosen based on elastic parameters, long term stability, ease of handling and molding. They can be stored long term to enable significant re-use without degradation. ${ }^{54,55}$ Some of the commercially available RTV silicones are discussed below: 
- SoftGel A-341C (Factor II Incorporated, AZ, USA): This material is a translucent, low viscosity RTV silicone gel that develops much of the same dimensional stability and non flowing characteristics of a solid silicone elastomers. The gel is used to produce healthy and cancerous tissues. Curing time is approx 1 hour at room temperature $\left(20-25^{\circ} \mathrm{C}\right) .{ }^{56}$

- DC 200 Silicone Fluid 50 cs (Dow Corning Corp. MI, USA): This material is used as thinning agent to be mixed with silicone gel to produce softer elastic properties of the cured material. This material is now available under a new product name i.e. Xiameter PMX $200 .^{57}$

- LSR-05 Silicone Elastomer (Factor II Incorporated, AZ, USA): This material is a 1:1 platinum cured translucent low viscosity RTV that cures in approximately 3 hours at room temperature. When cured, it yields hard elastic properties and can be used for fabrication of tumors and skin in appropriate proportions. ${ }^{58}$

- Elastosil P7600 RTV-2 A/B (Wacker Inc., AG, Germany): The cured silicone exhibits a similar elasticity as SoftGel A-341C, but with much higher internal damping. With Dow Corning Fluid 50 cs in 1:1 ratio, it exhibits very desirable elastic properties and can be used for phantoms with lower Young's modulus values. Vulcanization is approx 6-8 hours at room temperature or 1 hour at $100^{\circ} \mathrm{C} .{ }^{59}$

\section{II.C. Selected compositions for dynamic mechanical analysis}

Eight compositions were chosen to deliver a range of mechanical properties to best match the reported tissue characteristics ${ }^{1-4,39,41-43}$ and based on obtained results; phantoms for MRE and DIET imaging were developed. The compositions of silicone materials for DMA testing were as follows:
A: $\quad 100 \%$ SoftGel A-341C
B: $\quad 70 \%$ SoftGel A-341C $+30 \%$ DC 200 Silicone Fluid 50 cs
C: $\quad 50 \%$ SoftGel A-341C $+50 \%$ DC 200 Silicone Fluid 50 cs
D: $\quad 30 \%$ SoftGel A-341C + $70 \%$ DC 200 Silicone Fluid 50 cs
E: $\quad 100 \%$ LSR-05 Silicone Elastomer 


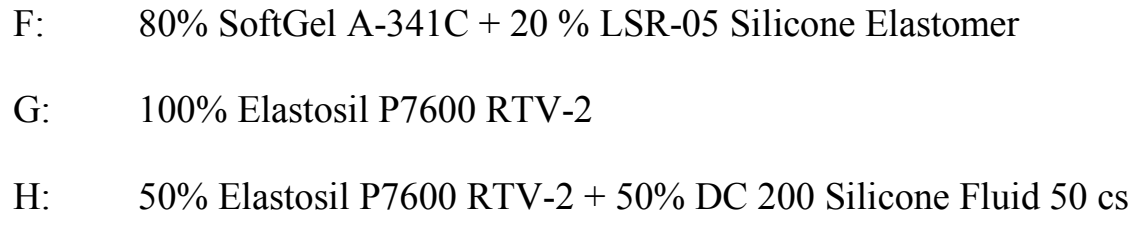

\section{II.D. Dynamic mechanical analysis}

Five cylindrical samples ( $8 \mathrm{~mm}$ thick, $28 \mathrm{~mm}$ diameter) from each composition using the same batch of silicone were cast for mechanical testing. The samples were tested in compression at room temperature. A Q-8000 dynamic mechanical analyzer (DMA) (TA instruments, New Castle, DE, USA) was used to test the samples dynamically over a range of $4-50 \mathrm{~Hz}$ (isothermally) at room temperature. Storage modulus $\left(E^{\prime}\right)$, loss modulus (E”) and damping ratios $\left(\zeta=\frac{E^{\prime \prime}}{E^{\prime}}\right)$ were obtained using a viscoelastic bar model:

$$
\bar{F}=\frac{A E^{*}}{t} \bar{X}
$$

155 where $\bar{F}$ is the measured force amplitude, $\bar{X}$ is the corresponding displacement amplitude, A is sample cross-section area, $\mathrm{t}$ is sample thickness and $E^{*}$ represents the complex valued viscoelastic Young's modulus $\left(E^{*}=E^{\prime}+i E^{\prime \prime}\right)$. The model equation assumes that transverse strains are negligible. In fact, because of constraints applied by the platens of the DMA, transverse strains in the sample are significant, so stiffness computed using Equation (1) will be higher than the actual sample stiffness. To account for this offset, the DMA stiffness estimates apply an empirical correction factor based on sample geometry, so that: 


$$
E^{*}=\frac{\bar{F} F_{e} t}{\bar{X} A}
$$

where $F_{e}$ is the clamping correction factor, determined by the DMA using Finite Element Analysis of the sample deformation. For a solid circular sample of thickness, $t=8 \mathrm{~mm}$, diameter, $\mathrm{D}=28 \mathrm{~mm}$, and Poisson's ratio, $v=0.45$ (assumed), $F e$ was calculated to be 0.5487 using the following relation: ${ }^{60,61}$

$$
F_{e}=\frac{1-1.999 * v+0.03745 *\left(\frac{t}{D}\right)+2.457 *\left(\frac{t}{D}\right)^{2}-1.244 *\left(\frac{t}{D}\right)^{3}}{1.009-1.814 * v+0.8257 * v^{2}-0.1303 *\left(\frac{t}{D}\right)+2.776 *\left(\frac{t}{D}\right)^{2}-1.461 *\left(\frac{t}{D}\right)^{3}}
$$

\section{II.E. DMA testing procedure}

The required preload was calculated to produce a $1 \%$ pre-strain based on the estimated modulus of the first sample of each composition.

a. The dynamic strain was set to $2 \%$, half of the peak-to-peak difference (i.e. strain $= \pm 2 \%$ ).

b. The 'Force Track' was set to $125 \%$, which means the DMA applies $125 \%$ of $2 \%$ extra preload to make sure the top plate does not lose contact with the top surface of the sample. In other words, approximately $3.5 \%$ pre-strain was applied, and then oscillated at $\pm 2 \%$.

c. The Young's modulus $E^{*}$ calculated according to Equation

d. (2) also accounts for clamping effects of the material surfaces as DMA applies an empirical correction factor to account for this. The modulus is calculated in terms of its real and imaginary components i.e. storage modulus $\left(E^{\prime}\right)$ loss modulus $\left(E^{\prime \prime}\right)$ and damping ratio $\left(\zeta=E^{\prime \prime} / E^{\prime}\right)$

e. The correction factor, $\mathrm{Fe}$, for the boundary conditions assumed a Poisson's ratio of 0.45 for these samples and the calculated $F e$ was 0.55 . 
f. One DMA measurement was recorded for each of the five samples and variation was gauged by using multiple samples rather that multiple measurements on the same sample. The coefficient of variation was calculated on the basis of accumulative mean, $\mu$, and standard deviation, $\sigma$, of all the five samples.

\section{II.F. Phantom molding}

\section{II.F.1. Design of mold}

A custom mold matching the shape of a human breast was constructed. The mold consisted of two assemblies; a cavity [Fig. 1(A)] and a core [Fig. 1(B)] such that there was a $1 \mathrm{~mm}$ gap between the two, when core seated in the cavity [Fig. 1(C)]. The core was used to mold a skin that can have a significant effect on the overall mechanical response of the phantom due to the compressive constraining traction it supplies, thus affecting the surface motion reconstruction in DIET. The mold was designed as a 3D CAD model and CNC milled out of aluminum.

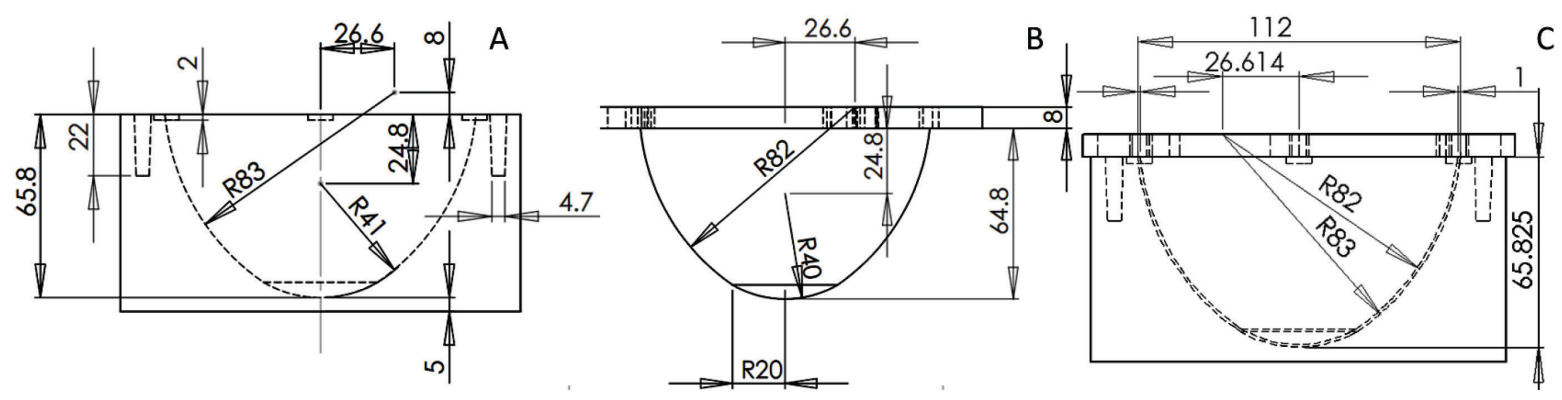

FIG.1. Drawings of phantom mold (A) cavity, (B) core and (C) complete assembly showing $1 \mathrm{~mm}$ gap between cavity and core

\section{II.F.2. Molding procedure}

195 Based on the results of DMA testing, a set of 13 anthropomorphic phantoms was developed for evaluation of DIET breast cancer screening, including one homogenous healthy (with no inclusion) and 12 heterogeneous phantoms with stiffer spherical inclusions (tumors) of various sizes $(5 \mathrm{~mm}$, $10 \mathrm{~mm}$, and 20mm) embedded at different depth wise locations (A, B, C and D) as shown in Figure 2(A, B \& C). B10 for example, shows a 10mm tumor at location B. A detailed description of these

200 locations is given in Table II. Each of these inclusions were positioned at 6-o'clock (feet side) when viewed from the front [Fig. 2(D)]. The MRE phantom was rectangular block shaped (dimensions: 90 
x $60 \times 60 \mathrm{~mm}^{3}$ ) having two rectangular block inclusions (dimensions: $30 \times 20 \times 20 \mathrm{~mm}^{3}$ ) with different elastic parameters.

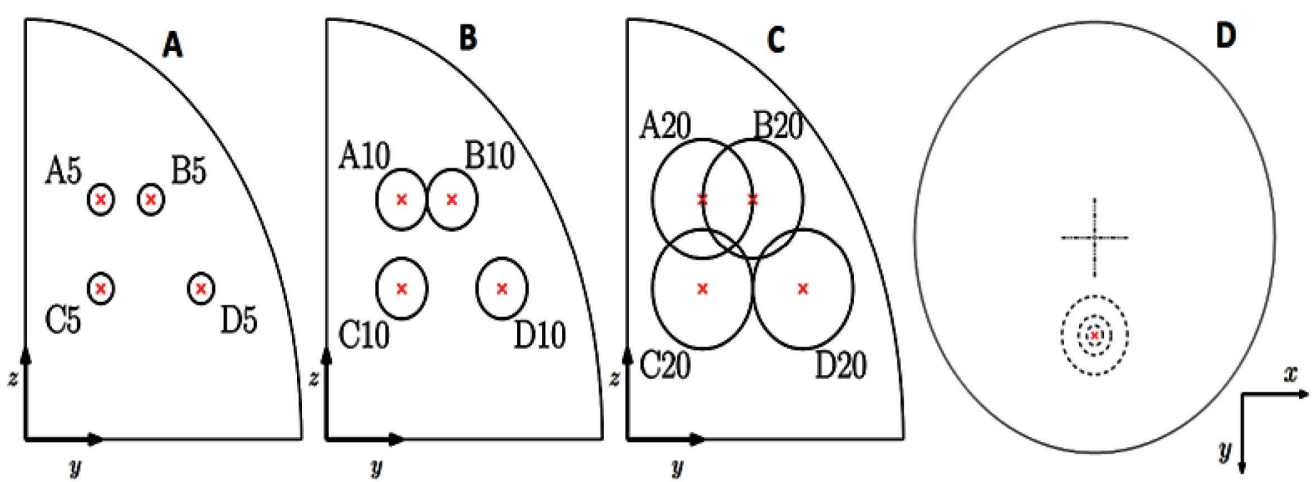

FIG. 2. Depth locations of inclusions (A) 5mm, (B) 10mm and (C) 20mm, and (D) 6-o' clock position when the breast is viewed from front.

Table II. Description of tumor location (depth) in heterogeneous breast phantoms

\begin{tabular}{ccc}
\hline \hline Location & $\begin{array}{l}\text { Distance from chest } \\
\text { muscle }(\mathrm{mm})\end{array}$ & $\begin{array}{l}\text { Distance from center of } \\
\text { the breast }(\mathrm{mm})\end{array}$ \\
\hline $\mathrm{A}$ & 40 & 15 \\
$\mathrm{~B}$ & 40 & 25 \\
$\mathrm{C}$ & 25 & 15 \\
$\mathrm{D}$ & 25 & 35 \\
\hline
\end{tabular}

Before starting to mold the DIET phantom, a release agent Silicone Star (Sta-Lube, Auckland, NZ) was thinly sprayed on the core so that it leaves the skin attached to the inner surface of the cavity during extraction. All mixing, stirring and curing were done at room temperature. The detailed procedure to create an anthropomorphic silicone breast phantom is described:

II.F.2.a. Skin: $\quad 50 \mathrm{~g}$ of silicone material were required to fabricate $1 \mathrm{~mm}$ of skin. The material was mixed per the composition chosen and few drops of coloration Suntan Fl-227 and White Fl-200 (Factor II - Factor II Incorporated, AZ, USA) was mixed to get the skin color. The mix was stirred in a plastic container, and de-aired in a vacuum chamber for 15 minutes. The material was then poured into the mold, the core was placed into the cavity and the material was 
left for curing for 2 hours at room temperature. For extraction the core was lifted gradually by tightening the jacking screws one by one, as shown in Figure 3(A). The skin was left attached to the inner surface of the cavity for subsequent molding of the other tissues.

II.F.2.b. Tumor: The silicone composition chosen for tumor was mixed in a disposable paper glass, stirred, de-aired and left for curing for one hour. After curing, the silicone was released and cut into spherical tumors of $5 \mathrm{~mm}, 10 \mathrm{~mm}$, and $20 \mathrm{~mm}$ diameter. The tumor was suspended in the mold using a thin wire at the desired location and the material for the adipose tissue was poured into the mold as shown in Figure 3(B).

II.F.2.c. Adipose tissue: $\quad 300 \mathrm{~g}$ of material chosen for adipose was mixed in the recommended ratios, and de-aired for 15 minutes. The material was slowly poured into the cavity withholding the prefabricated skin, to avoid formation of air bubbles. This material was enough to fill the mold cavity, leaving a $10 \mathrm{~mm}$ gap at the top for subsequent molding of the chest muscle as shown in Figure 3(C). At room temperature, this material took almost 3 hours to cure.

II.F.2.d. Pectoral Muscle: After the material in the mold was properly cured, the wire holding the tumor was slowly pulled and a circular acrylic disc with three attached bolts [Fig. 3(D)] was placed on top of the material. This disc was meant to mimic the effect of the rib cage, presenting a stiff backing and the bolts helped in mounting the phantoms during DIET imaging. $88 \mathrm{~g}$ of a final silicone compound was poured around the disc as shown in Figure 3(E) and left at room temperature to cure for one hour.

Extreme care was exercised while extracting the phantom to avoid any damage to the skin. Individual silicone components were well bound to each other and allowed robust handling of the phantom as a single unit [Fig. 3(F)]. 


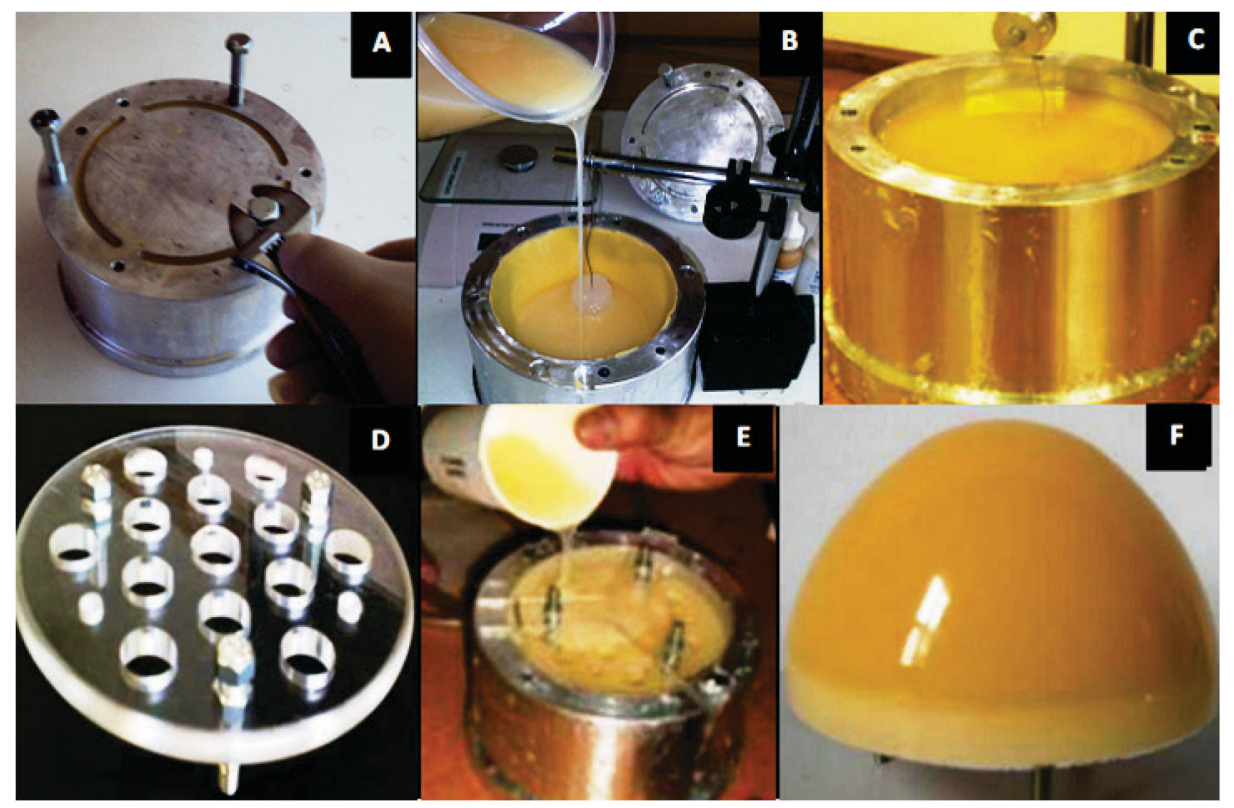

FIG.3. Molding procedure of silicone breast phantoms: (A) process of core extraction (B) procedure of placing a stiff tumor into the healthy tissue, (C) cured healthy tissue, (D) circular disc with bolts, (E) method of applying a circular disk during molding of pectoral muscle, $(\mathrm{F})$ the molded breast phantom.

\section{II.F.3. Phantom imaging}

II.F.3.a. DIET imaging: Red, green and blue papers were cut with a scissors in the ratio of 1:2:2 respectively, in the smallest possible size $(1 \mathrm{~mm} \times 1 \mathrm{~mm})$ to form fiducial markers and 1200-1500 of these markers were applied on each phantom to cover the whole surface. Silicone provided the natural adhesion. The markers purpose of applying these markers was to ensure robust motion tracking when optically imaged with the DIET system. ${ }^{28,29,32,33,47,48,62-64}$ During the imaging procedure each one of these 13 phantoms, was individually mounted on a metallic plate $(200 \mathrm{~mm} \times 150 \mathrm{~mm})$ and hung pendant through a hole in the DIET clinical prototype. The phantom was vibrated using harmonic sinusoidal actuation of $0.5 \mathrm{~mm}$ amplitude, over a range of $10-50 \mathrm{~Hz}$ at a step size of $1 \mathrm{~Hz}$. The motion was frozen at every 36 degrees and 10 optical images at each frequency were obtained using an array of 5 digital cameras in the device. The motion was reconstructed in $3 \mathrm{D}^{32,33,62,63}$ and a spherical coordinate system was defined to describe the location of the applied fiducial markers. The whole breast phantom was discretized into 126 segments to account for uneven marker coverage ${ }^{49}$ and Bode diagrams of the frequency response (with respect to the actuation) were obtained covering the first and second resonant frequencies for each segment. The obtained resonant frequencies were compared 
across the surface to observe differences in behavior. To assess statistical significance of the differences observed, the two-sample t-test was used to reject the hypothesis that two areas were similar in the resonance behavior. A significance threshold of $\alpha=0.01$ was chosen, indicating a $99 \%$ confidence interval. The hypothesis tested was that the area of interest has the same or different resonance compared to the rest of the surface..$^{49,50}$

265 II.F.3.b. MR imaging: MRE was performed on the MRE phantom using a Philips Achieva 3T MRI system. A piezoelectric actuator driven at $100 \mathrm{~Hz}$ was used to generate harmonic motion, which was measured in all three coordinate directions using an echo planar imaging sequence with added motion sensitizing gradients. Full volume measurements in all three motion directions were taken using $2 \mathrm{~mm}$ isotropic voxels. A subzone based algorithm was then used to estimate the viscoelastic mechanical property distribution by iteratively updating an estimate of the properties to minimize the difference between the displacements predicted by a computational model and the measurements. ${ }^{65}$ 


\section{IIII. Results}

\section{III.A. Elastic properties of tested materials}

275 Accumulative mean, $\mu$, of storage modulus, $E^{\prime}$, and damping ratios $\left[\tan \delta=\frac{\text { loss modulus }\left(E^{\prime \prime}\right)}{\text { Storage } \text { modulus }\left(E^{\prime}\right)}\right]$, denoted by $\zeta$, were obtained for all five samples from each composition using the DMA measurements. E' ranged from $2 \mathrm{kPa}(\mathrm{D})$ to $570 \mathrm{kPa}(\mathrm{E})$, and $\zeta$ ranged from $0.03(\mathrm{~B})$ to $0.56(\mathrm{H})$. Samples from Composition D were the softest and showed abrupt anomalous behavior at high frequencies, mainly due to its very low stiffness and machine resonance. To show the extent of variability, standard deviations, $\sigma$, were obtained from the DMA measurements and coefficients of variation $\left(C_{v}=\frac{\sigma}{\mu}\right)$ were calculated for all compositions and plotted along with mean, $\mu$, values. Coefficients of variation $\left(C_{v}=\frac{\sigma}{\mu}\right)$ in most of the cases were reasonably low except for composition D, which may be attributed to experimental errors. The DMA results of storage modulus, $E^{\prime}$, are shown in Figure 4 (A \& B) and damping ratios, $\zeta$, are shown in Figure 5 (A \& B).

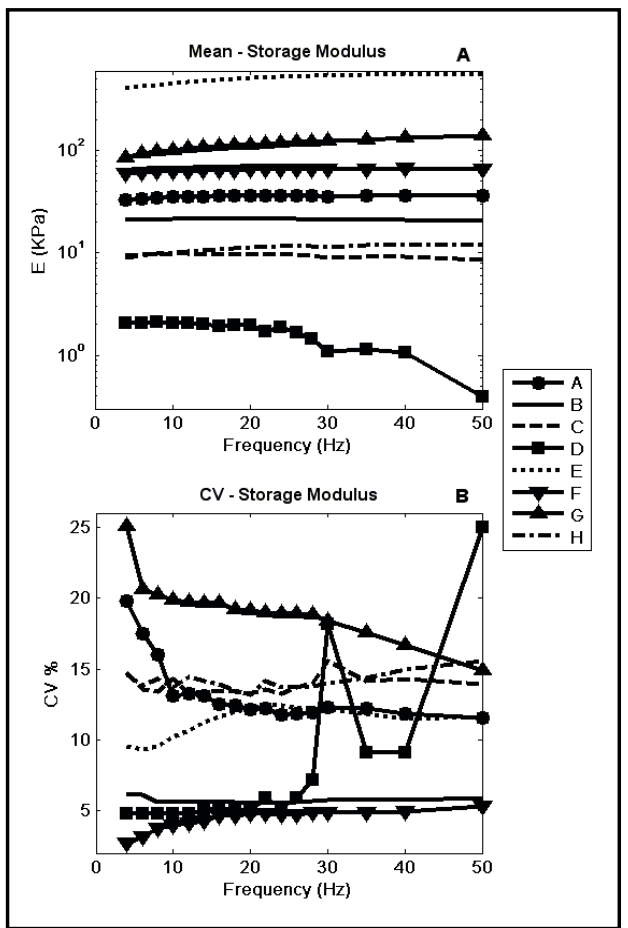

FIG. 4. (A) Mean values, $\mu$, and (B) Coefficient of variation $(C v=\sigma / \mu)$ of storage Modulus, $E$, 


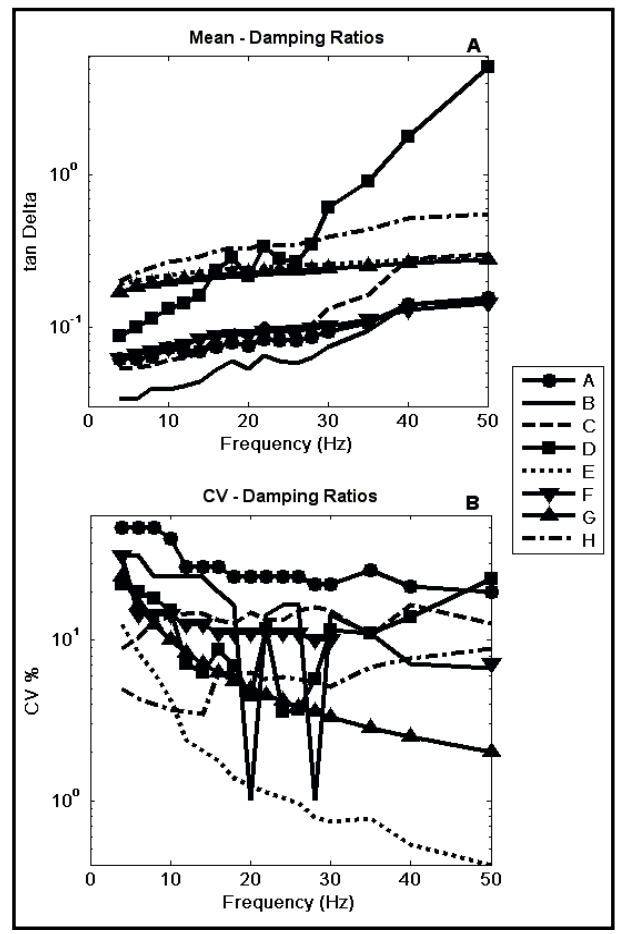

FIG.5. (A) Mean values, $\mu$, and (B) coefficient of variation $(C v=\sigma / \mu)$ of damping ratios, $\zeta$.

\section{III.B. Phantom application}

\section{III.B.1. DIET results}

Based on the DMA results, the compositions chosen for different tissues in DIET phantom and their elastic parmeters are shown in Table III.

Table III: Materials used for mimicking different tissues in DIET anthropomorphic breast phantoms

\begin{tabular}{|c|c|c|c|c|}
\hline Tissue & Composition & Material & $\begin{array}{l}\text { Storage } \\
\text { Modulus, } E \\
(\mathrm{kPa})\end{array}$ & $\begin{array}{l}\text { Damping } \\
\text { ratios, } \zeta\end{array}$ \\
\hline Skin & $\mathrm{F}$ & $\begin{array}{l}80 \% \text { SoftGel A-341C + } 20 \% \text { LSR- } 05 \\
\text { Silicone Elastomer }\end{array}$ & 60 & 0.09 \\
\hline Tumor & A & $100 \%$ SoftGel A-341C & 36 & 0.08 \\
\hline Adipose & $\mathrm{C}$ & $\begin{array}{l}50 \% \text { SoftGel A-341C + } 50 \% \text { DC } 200 \\
\text { Silicone Fluid } 50 \mathrm{cs}\end{array}$ & 9.5 & 0.06 \\
\hline $\begin{array}{l}\text { Pectoral } \\
\text { Muscle }\end{array}$ & A & $\begin{array}{l}100 \% \text { SoftGel A-341C embedded into } \\
\text { a circular acrylic disc with holes }\end{array}$ & 36 & 0.08 \\
\hline
\end{tabular}


The breast phantoms were imaged using a DIET clinical prototype $e^{28,48}$ and the surface motion was reconstructed in 3D. The whole surface was discretized into 126 segments and the frequency response was studied in each segment to analyse variations in first and second natural frequencies. The locations of the resonant frequencies were noticeably different around the tumor location, as 300 compared to the healthy area, both in terms of manitude [Fig. 6(A)] and phase [Fig. 6(B)]. The solid line in Figure 6 (A \& B) corresponds to Bode diagram of a surface segment away from the inclusion area and the dotted line corresponds to vibration of a surface segment close to the inclusion area. A shift in the first and second peak was clearly observed in areas closer to inclusion as compared to areas away from inclusion. Moreover, the damping in the inclusion areas was clearly higher as compared to the damping in the areas away from inclusion. The two-sample t-test was used and $p$ values were obtained to give indication of the difference in the observed metric in one segment, compared to the remaining breast surface. To analyse these differences across the breast, the results of t-test were compared around the surface of the phantoms. Figure 7 (A \& B) show results of the t-test applied on a healthy phantom (with no inclusion) and a phantom with $20 \mathrm{~mm}$ tumor at location B

310 (Table II) in 6-0'clock position. The red area (value 1) shows segments where the null hypothesis was rejected, indicating an abrupt change in the natural frequencies with a $99 \%$ of confidence level. ${ }^{49}$ 


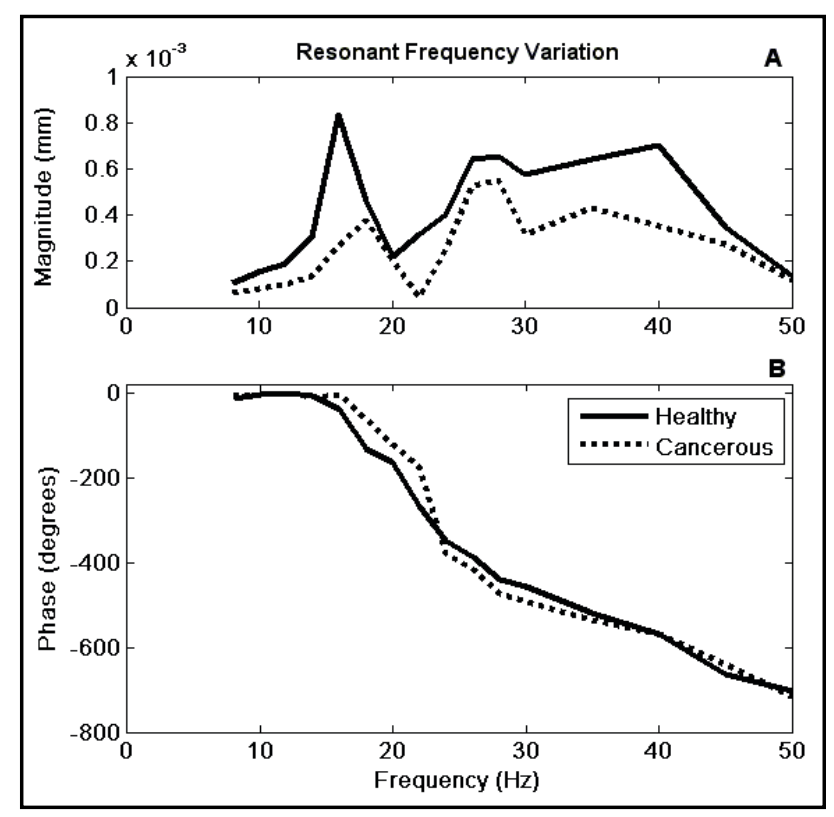

FIG.6. Bode plot of healthy (solid) and tumors data set (dotted) showing shift in the natural frequencies both in terms of magnitude of surface vibration (A) and Phase (B)
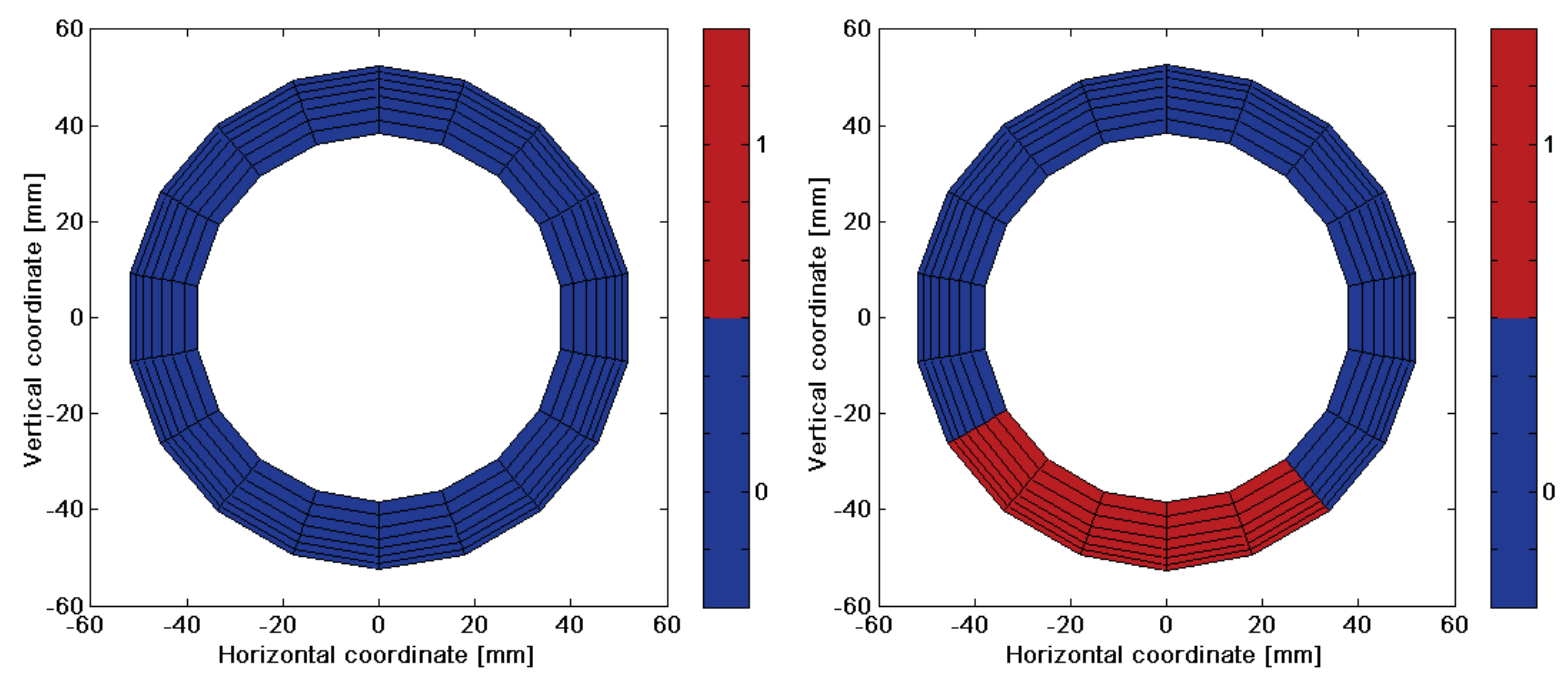

FIG.7. Result of $t$-test for phantoms (A) healthy and (B) having a 20mm tumor at 6 o' clock. The thresholded outcome 1-p hypothesis rejected (red) or accepted (blue) is shown in each case, indicating an abrupt change in the natural frequencies and thus a stiffer inclusion at the red area.

To show the range of possibilities in mechanical elastic properties testing, an MRE phantom was made in a rectangular shape. Two inclusions, one with lower and one with higher stiffness than the background were embedded in this phantom to assess the possibility of detecting a softer inclusion in 
the harder background. The details compositions used for mimicking background and tumors for the

MRE phantom are given in Table IV.

Table IV: Materials used for mimicking background and inclusions in the MRE phantom

\begin{tabular}{|c|c|c|c|c|}
\hline Tissue & Composition & Material & $\begin{array}{c}\text { Storage } \\
\text { Modulus, } E \\
(\mathrm{kPa})\end{array}$ & $\begin{array}{l}\text { Damping } \\
\text { ratios, } \zeta\end{array}$ \\
\hline Background & $\mathrm{B}$ & $\begin{array}{l}70 \% \text { SoftGel A-341C }+30 \% \text { DC } \\
200 \text { Silicone Fluid } 50 \mathrm{cs}\end{array}$ & 21 & 0.05 \\
\hline Tumor1 & $\mathrm{A}$ & $100 \%$ SoftGel A-341C & 36 & 0.08 \\
\hline Tumor2 & $\mathrm{C}$ & $\begin{array}{l}50 \% \text { SoftGel A-341C }+50 \% \text { DC } \\
200 \text { Silicone Fluid } 50 \mathrm{cs}\end{array}$ & 9.5 & 0.06 \\
\hline
\end{tabular}

MRE reconstructions of this phantom also distiguished inclusions from the background and clearly indicated the presence of both inclusions in the phantom, in the observed metrics, as shown in Figure $8(\mathrm{~A}-\mathrm{D})$.
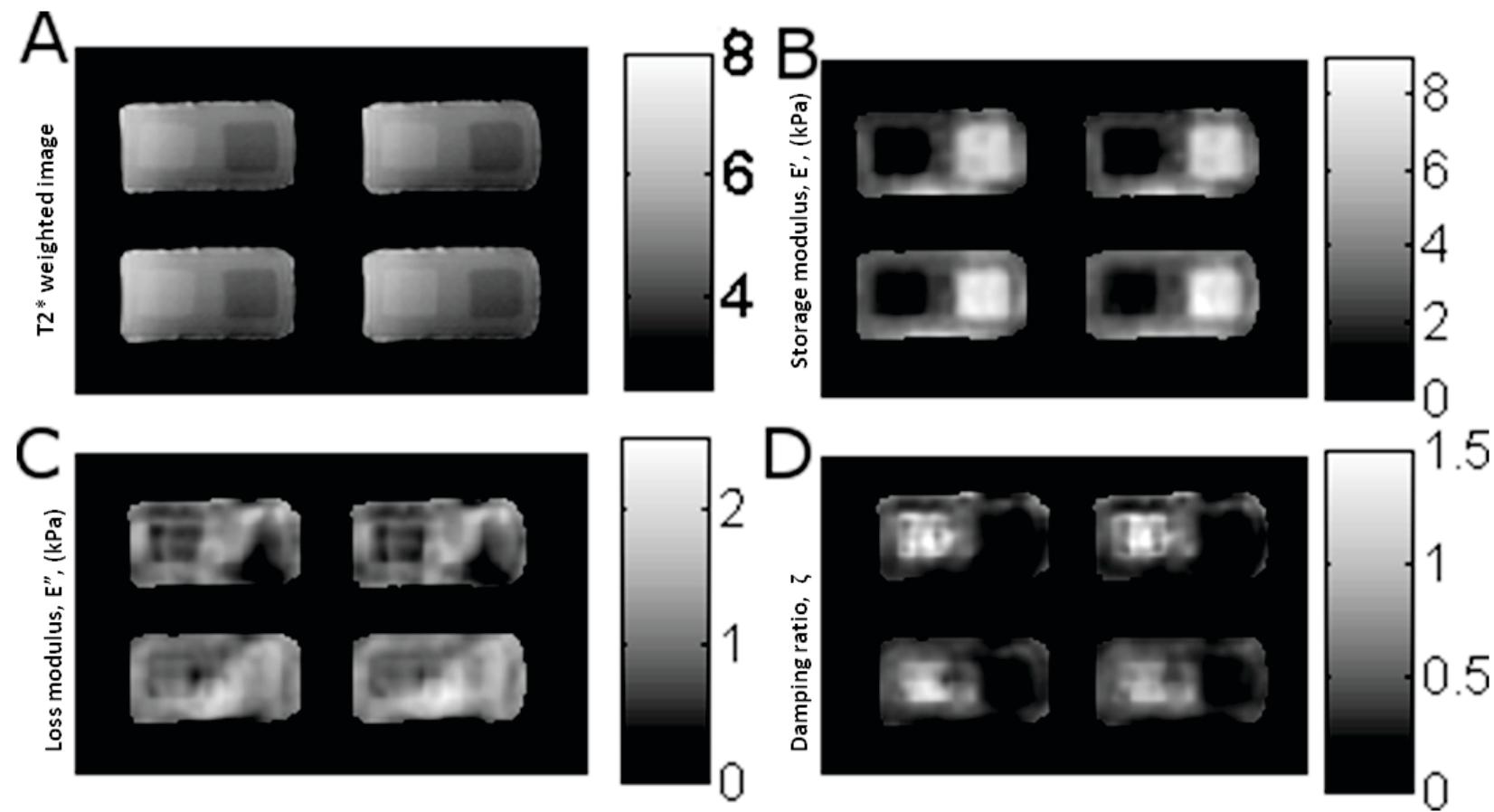

FIG.8. MRE Reconstruction of a phantom with 'composition-B' as background and two inclusions: 'composition-C' on the left and 'composition-A' on the right. A subset of slices from the full volume data read shown: (A) is a T2* weighted image, (B) is the storage modulus $(\mathrm{kPa}),(\mathrm{C})$ is the loss modulus $(\mathrm{kPa})$ and $(\mathrm{D})$ is the damping ratio. 


\section{Discussion}

This study was conducted to estimate elastic properties of various silicone materials to allow various components of human breast tissue to be mimicked so that an anthropomorphic breast phantom may be fabricated for testing of elastographic imaging modalities. It is important that a phantom has comparable elastic properties to give a relevant evaluation of an imaging system.

The dynamic mechanical testing proved to be vital in assessing the stiffness parameters of various silicone elastomers and providing benchmarking towards development of a breast phantom. The DMA results are consistent and provide a good match to some reported human breast tissue elasticity and breast phantoms previously used for research. There were some abrupt anomalies observed over higher frequencies with the very soft samples of composition D (30\% SoftGel A-341C + 70\% DC$200, E^{\prime}=0.4-2.1 \mathrm{kPa}$ ) which may be attributed to experimental errors. The machine resonance at higher frequencies might have dominated the elastic forces of the softest material due to inertial forces, leading to significant errors in the stiffness estimates of composition D.

These results provide a basis for a wide selection of materials to mimic skin, healthy and cancerous tissues in the breast. Composition C $\left(50 \%\right.$ SoftGel A-341C $+50 \%$ DC-200, $\left.E^{\prime}=9 \mathrm{kPa}, \zeta=0.06\right)$ was chosen to replicate the combined effect of the fatty and fibro-glandular tissues present in the female human breast, as a 3 composite silicone phantom that captures both tissues separately is not entirely feasible. Composition A (100\% SoftGel A-341C, $\left.E^{\prime}=35 \mathrm{kPa}, \zeta=0.08\right)$ was used to mimic cancerous tumors in the DIET anthropomorphic breast phantom, yielding a stiffness contrast of almost 4x.

DMA results for storage modulus, $E$ ', for all the eight compositions ranged from 2 to $570 \mathrm{kPa}$, covering almost the whole range of elastic properties of various healthy and diseased human breast tissues reported by the previous studies. The minimum value of Young's modulus ( $\left.E^{*}=E^{\prime}+i E^{\prime \prime}\right)$ for adipose tissue, has been reported by Liang et al. ${ }^{43}$ as $0.42 \mathrm{kPa}$ and the maximum value for carcinoma $460 \pm 178 \mathrm{kPa}$ at $20 \%$ compression has been reported by Krouskop et al. ${ }^{1}$ However, Saravazyan et al. ${ }^{4}$ 
reported $100-5000 \mathrm{kPa}$ for a palpable nodule, which is exceptionally high compared to other studies. These differences can be attributed to different measurement techniques adopted by these researchers.

This study establishes a broad range of values that can be obtained using the commonly available silicone elastomers. More experiments by varying the ratios of the mixtures can further increase the selection of the required composition to mimic a range of tissues. Other compositions with higher damping can also be chosen, depending upon the required application and elastographic modality being used. DMA results may prove to be useful for developing phantoms of other human organs depending upon the elasticity requirements. Two different types of phantoms were constructed and tested using the DIET and MRE, and results show a meaningful tissue contrast required to distinguish between a healthy and cancerous tissues.

It was noted that the damping ratios of the chosen materials were relatively low as compared to the actual human breast, and it would be interesting to see the results of phantoms developed with a higher damping material as a future study. Composition H (50\% Elastosil P7600 RTV-2 + 50\% DC 200 Silicone Fluid $50 \mathrm{cs}$ with $E^{\prime}=8.9-10.2 \mathrm{kPa}$ and $\zeta=0.20-0.56$ ) bears strong candidature for a future study. The hyperelatstic behaviour of breast tissue has not been given due emphasis in this research and a future study with a main focus on matching the nonlinear elasticity properties of the breast bears a great potential.

Moreover, as the main focus of this research was to develop breast phantoms for optical imaging required for evaluation of DIET technology, consideration was not given to match the magnetic properties of the tissue mimicking materails. Thus, while the main goal of matching fundamental stiffness moduli is met, there are avenues for improvement.

The anthropomophic breast phantoms developed for evaluation of DIET breast cancer screening system, proved to be easy to handle. Phantoms constructed two years ago are still in good condition and remain in use for DIET in vitro trials. 


\section{Conclusion}

Eight compositions were mechanically tested using DMA, revealing a range of material properties spanning over $E^{\prime}=2-570 \mathrm{kPa}$. Anthropomorphic breast phantoms comprised of tissue mimicking skin, adipose, cancerous tumor and pectoral muscles were constructed, and tested using a DIET system. During the modal analysis of these phantoms, a significant shift in first and second resonance

390 frequencies was observed in the inclusion areas. To assess the range of possibilities a silicone phantom with two inclusions of different elastic parameters was put to trial using an MRE system. This phantom showed intrinsic contrast in the $\mathrm{T} 2 *$ weighted images.

The mechanical property images also clearly showed stiffness contrast between the materials of background and the two inclusions. Varying stiffness properties can be achieved by altering the ratios

395 of the investigated materials for mimicking tissues of other human organs. A dedicated study to match the magnetic and nonlinear properties of the tissue mimicking materials chosen for breast phantom development is intended and bears a great potential.

Overall, the anthropomorphic breast phantoms seemed to show a stable response in handling and the findings of this study render the developed breast phantoms useful for in vitro evaluation of DIET and 400 MRE. 


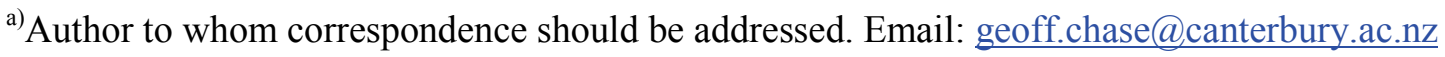

${ }^{1}$ T. A. Krouskop, T. M. Wheeler, F. Kallel, B. S. Garra and T. Hall, "Elastic moduli of breast and prostate tissues under compression," Ultrasonic Imaging 20 (4), 260-274 (1998).

${ }^{2}$ A. Samani, J. Zubovits and D. Plewes, "Elastic moduli of normal and pathological human breast tissues: An inversion-technique-based investigation of 169 samples," Phys. Med. Biol. 52 (6), 15651576 (2007).

${ }^{3}$ A. Samani, J. Bishop, C. Luginbuhl and D. Plewes, "Measuring the elastic modulus of ex vivo small tissue samples," Phys. Med. Biol. 52, 1565 - 1576 (2003).

${ }^{4}$ A. P. Sarvazyan, A. R. Skovoroda, S. A. Emelianov, J. B. Fowlkes, J. G. Pipe, R. S. Adler, R. B.

410 Buxton and P. L. Carson, "Biophysical bases of elasticity imaging," Acoustical Imaging 21, 223-240 (1995).

${ }^{5}$ K. C. Chu and B. K. Rutt, "Polyvinyl alcohol cryogel: An ideal phantom material for MR studies of arterial flow and elasticity," Magnetic Resonance in Medicine 37 (2), 314-319 (1997).

${ }^{6}$ V. Egorov, S. Tsyuryupa, S. Kanilo, M. Kogit, and A. P. Sarvazyan, "Soft tissue elastometer," 415 Medical Engineering and Physics 30 (2), 206-212 (2008).

${ }^{7}$ E. L. Madsen, G. R. Frank, M. A. Hobson, H. Shi, J. Jiang, T. Varghese, and T. J. Hall, "Spherical lesion phantoms for testing the performance of elastography systems," Phys. Med. Biol. 50 (24), 5983 (2005).

${ }^{8}$ T. J. Hall, Y. Zhu and C. S. Spalding, "In vivo real-time freehand palpation imaging," Ultrasound Med. Biol. 29 (3), 427-435 (2003).

${ }^{9}$ E. L. Madsen, M. A. Hobson, G. R. Frank, H. Shi, J. Jiang, T. J. Hall, T. Varghese, M. M. Doyley, and J. B. Weaver, "Anthropomorphic breast phantoms for testing elastography systems," Ultrasound Med. Biol. 32 (6), 857-874 (2006).

${ }^{10}$ A. L. McKnight, J. L. Kugel, P. J. Rossman, A. Manduca, L. C. Hartmann and R. L. Ehman, "MR elastography of breast cancer: Preliminary results," American Journal of Roentgenology 178 (6), 1411-1417 (2002).

${ }^{11}$ R. Muthupillai, D. J. Lomas, P. J. Rossman, J. F. Greenleaf, A. Manduca and R. L. Ehman, "Magnetic resonance elastography by direct visualization of propagating acoustic strain waves," Science (New York, N.Y.) 269 (5232), 1854-1857 (1995).

$430{ }^{12}$ R. Muthupillai, P. J. Rossman, D. J. Lomas, J. F. Greenleaf, S. J. Riederer and R. L. Ehman, "Magnetic resonance imaging of transverse acoustic strain waves," Magn. Reson. Med. 36 (2), 266274 (1996).

${ }^{13}$ K. J. Parker, M. M. Doyley and D. J. Rubens, "Imaging the elastic properties of tissue: The 20 year perspective,” Phys. Med. Biol. 56 (1), R1 (2011).

$435{ }^{14}$ D. B. Plewes, I. Betty, S. N. Urchuk and I. Soutar, "Visualizing tissue compliance with MR imaging," Journal of Magnetic Resonance Imaging 5 (6), 733-738 (1995). 
${ }^{15}$ K. Siegmann, T. Xydeas, R. Sinkus, B. Kraemer, U. Vogel and C. Claussen, "Diagnostic Value of MR elastography in addition to contrast-enhanced MR imaging of the breast-Initial Clinical Results,” European Radiology 20 (2), 318-325 (2010).

$440 \quad{ }^{16}$ R. Sinkus, M. Tanter, S. Catheline, J. Lorenzen, C. Kuhl, E. Sondermann and M. Fink, "Imaging anisotropic and viscous Properties of breast tissue by magnetic resonance-elastography," Magn. Reson. Med. 53 (2), 372-387 (2005).

${ }^{17}$ J. B. Weaver, E. E. Van Houten, M. I. Miga, F. E. Kennedy and K. D. Paulsen, "Magnetic resonance elastography using 3D gradient echo measurements of steady-state motion," Med. Phys. 28 (8), 1620$1628(2001)$.

${ }^{18} \mathrm{Y}$. Zhu and T. J. Hall, "A modified block matching method for real-time freehand strain imaging," Ultrasonic Imaging 24 (3), 161-176 (2002).

${ }^{19}$ D. B. Kopans, Breast Imaging, 3rd ed. (Lippincott Williams \& Wilkins, Philadelphia, 2007).

${ }^{20}$ S. McDonald, D. Saslow and M. H. Alciati, "Performance and reporting of clinical breast examination: A review of the literature," CA: A Cancer Journal for Clinicians 54 (6), 345-361 (2004).

${ }^{21}$ A. Evans, P. Whelehan, K. Thomson, D. McLean, K. Brauer, C. Purdie, L. Jordan, L. Baker, and A. Thompson, "Quantitative shear wave ultrasound elastography: Initial experience in solid breast masses," Breast Cancer Research C7 - R104 12 (6), 1-11 (2010).

${ }^{22}$ W. Khaled, S. Reichling, O. T. Bruhns and H. Ermert, "Ultrasonic strain imaging and reconstructive elastography for biological tissue,” Ultrasonics 44, Supplement (0), e199-e202 (2006).

${ }^{23}$ K. Nightingale, M. S. Soo, R. Nightingale and G. Trahey, "Acoustic radiation force impulse imaging: In vivo demonstration of clinical feasibility," Ultrasound Med. Biol. 28 (2), 227-235 (2002).

${ }^{24}$ J. Ophir, I. Cespedes, B. Garra, H. Ponnekanti, Y. Huang and N. Maklad, "Elastography: Ultrasonic imaging of tissue strain and elastic modulus in vivo," European Journal of Ultrasound 3 (1), 49-70 460 (1996).

${ }^{25}$ A. C. Sharma, M. S. Soo, G. E. Trahey and K. R. Nightingale, "Acoustic radiation force impulse imaging of in vivo breast masses," presented at the Ultrasonics Symposium, 2004 IEEE, 2004.

${ }^{26} \mathrm{M}$. Doyley and J. Weaver, "Magnetic resonance elastography: Experimental validation and performance optimization," Alternative Breast Imaging (Springer US, 2005), 778, pp. 69-83.

${ }^{27}$ A. Manduca, T. E. Oliphant, M. A. Dresner, J. L. Mahowald, S. A. Kruse, E. Amromin, J. P. Felmlee, J. F. Greenleaf and R. L. Ehman, "Magnetic resonance elastography: Non-invasive mapping of tissue elasticity," Med. Image Anal. 5 (4), 237-254 (2001).

${ }^{28}$ T. Lotz, A. Kashif, S. Feng, P. Biret, Y. Denais, D. Lottin, L. Maillard, T. Tirschler and J. G. Chase, "A clinical prototype of the digital image elasto tomography breast cancer screening system," 470 presented at the Proc 5th International Conference on Bioinformatics and Biomedical Engineering (iCBBE), Wuhan, China, 2011.

${ }^{29}$ T. Lotz, P. D. Simpson, D. Stocker, C. E. Hann, and J. G. Chase, "In vitro evaluation of surface based non-invasive breast cancer screening with digital image based elasto tomography (DIET)," 
presented in 32nd Annual International Conference of the IEEE Engineering in Medicine and Biology Society (Buenos Aires, Argentina, 2010).

${ }^{30}$ A. Peters, A. Milsant, J. Rouze, L. E. Ray, J. G. Chase, and E. E. Van Houten, "Digital-image based elasto-tomography: Proof of concept studies for surface based mechanical property reconstruction," JSME International Journal 47 (4), 1117-1123 (2004).

${ }^{31}$ A. Peters, S. Wortmann, R. B. Elliot, M. Staiger, J. G. Chase and E. E.Van Houten, "Digital image480 based elasto-tomography: First experiments in surface based mechancial property estimation of gelatine phantoms,” JSME International Journal 48 (4), 562-569 (2005).

${ }^{32}$ R. G. Brown, C. E. Hann, J. G. Chase, and L. E. Ray, "Discrete colour-based euclidean-invariant signatures for feature tracking in a DIET breast cancer screening system," presented in SPIE Medical Imaging (SPIE, San Diego, USA, 2007), Vol. 6511.

$485{ }^{33}$ R. G. Brown, C. E. Hann, and J. G. Chase, "Vision-based 3D surface motion capture for the DIET breast cancer screening system," International Journal of Computer Applications in Technology 39 (1), 72-78 (2010).

${ }^{34}$ A. Peters, J. G. Chase, and E. E. Van Houten, "Digital image elasto-tomography: Combinatorial and hybrid optimization algorithms for shape-based elastic property reconstruction," IEEE trans. Bio-med. 490 Eng. 55 (11), 2575-2583 (2008).

${ }^{35}$ H.-U. Berger, C. E. Hann, J. G. Chase, R. L. Broughton and E. E. Van Houten, "Boundary element methods in elastography: A first explorative study," presented at the SPIE Medical Imaging, San Diego, USA, 2007.

${ }^{36}$ C. U. Devi, R. S. B. Chandran, R. M. Vasu and A. K. Sood, "Measurement of visco-elastic properties of breast-tissue mimicking materials using diffusing wave spectroscopy," J. Biomed. Optics 12 (3), 034035-034035 (2007).

${ }^{37}$ Y.C. Fung, "In biomechanics - Mechanical properties of living tissues," Springer, New York 2nd Edition (1993).

${ }^{38}$ K. R. Nightingale, R. W. Nightingale, M. L. Palmeri and G. E. Trahey, "A finite element model of 500 remote palpation of breast lesions using radiation force: Factors affecting tissue displacement," Ultrasonic Imaging 22 (1), 35-54 (2000).

${ }^{39}$ V. Egorov and A. P. Sarvazyan, "Mechanical imaging of the breast," IEEE Trans. Med. Imag. 27 (9), 1275-1287 (2008).

${ }^{40}$ A. Samani and D. Plewes, "An inverse problem solution for measuring the elastic modulus of intact ex vivo breast tissue tumours," Phys. Med. and Biol. 52 (5), 1247-1260 (2007).

${ }^{41}$ R. Sinkus, M. Tanter, T. Xydeas, S. Catheline, J. Bercoff, and M. Fink, "Viscoelastic shear properties of in vivo breast lesions measured by MR elastography," Magn. Reson. Imag. 23 (2), 159165 (2005).

${ }^{42}$ A. Srivastava, Y. Verma, K. D. Rao, P. K. Gupta, "Determination of elastic properties of resected 510 human breast tissue samples using optical coherence tomographic elastography," Strain 47 (1), 75-87 (2011). 
${ }^{43}$ X. Liang, A. L. Oldenburg, V. Crecea, S. Kalyanam, M. F. Insana and S. A. Boppart, "Modeling and measurement of tissue elastic moduli using optical coherence elastography," Proc. SPIE 6858, 685803 (2008), DOI:10.1117/12.760779

$515{ }^{44}$ A. Peters, J. G. Chase, and E. E. Van Houten, "Digital image elasto-tomography: Mechanical property estimation of silicone phantoms,” Med. Biol. Eng. Comput. 46 (3), 205-212 (2008).

${ }^{45}$ A. Samani and D. Plewes, "A method to measure the hyperelastic parameters of ex vivo breast tissue samples," Phys. Med. Biol. 49 (18), 4395 (2004).

${ }^{46}$ K. Nightingale, S. McAleavey and G. Trahey, "Shear-wave generation using acoustic radiation

520 force: in vivo and ex vivo results," Ultrasound Med. Biol. 29 (12), 1715-1723 (2003)

${ }^{47}$ R. G. Brown, J. G. Chase and C. E. Hann, "A pointwise smooth surface stereo reconstruction algorithm without correspondences,” Imag. Vision Comput. 30 (9), 619-629 (2012).

${ }^{48}$ J. G. Chase, A. Peters, T. F. Lotz and L. A. Ray, "Use of surface motion to identify mechanical properties of biological tissue," United States Patent Application, US 2009/0216131 A1, 27 Aug 2009.

${ }^{49}$ T. Lotz, A. M. W. Heeren, A. Kashif and J. G. Chase, "Elastographic tissue characterisation by separate modal analysis with a digital image elasto tomography (DIET) breast cancer screening system," presented in 9th IASTED Int Conf on Biomedical Engineering (BioMed 2012) (Innsbruck, Austria, 2012).

$530{ }^{50}$ T. F. Lotz, N. Muller, C. E. Hann, and J. G. Chase, "Minimal elastographic modeling of breast cancer for model based tumor detection in a digital image elasto tomography (DIET) system," Med. Imag. 2011: Computer-Aided Diagnosis 7963 796322-796322-796326 (2011).

${ }^{51}$ R. S. Hamas, "The comparative dimensions of round and anatomical saline-filled breast implants," Aesthetic Surgery Journal 20 (4), 281-290 (2000).

$535{ }^{52}$ R. S. Hamas, "The postoperative shape of round and teardrop saline-filled breast implants," Aesthetic Surgery Journal 19 (5), 369-374 (1999).

${ }^{53}$ M. Nipshagen, W. Beekman, D. Esmé and J. Becker, "Anatomically shaped breast prosthesis in vivo: A change of dimension," Aesthetic Plastic Surgery 31 (5), 540-543 (2007).

${ }^{54}$ A. Colas, "Silicone: Prepration, properties, and performance," Technical Report, Dow Corning Life 540 Sciences, Available at:

http://www.dowcorning.com/applications/search/default.aspx?Ntx=mode+MatchAllPartial|mode+Mat chAllPartial\&Ntk=ContentSearch en $\mid$ ContentSearch en\&Nty=1\&DCCD $=$ CONTENT\&WT.svl=1\&N $\underline{\mathrm{tt}=\text { Silicone }+ \text { Prepration } \mid+54+\mathrm{A}+\text { Colas }+ \text { Silicone }+ \text { Prepration }+ \text { Properties }+ \text { and }+ \text { Performancd }+2005+\& D}$ $\mathrm{CCTC}=$ true $\& N=0 \& D C C T=$ CONTENT2005

$545{ }^{55}$ H. Homma, C. L. Mirley, J. Ronzello and S. A. Boggs, "Field and laboratory aging of RTV silicone insulator coatings," IEEE Trans. Power Delivery 15 (4), 1298-1303 (2000).

${ }^{56}$ Factor II, Incorporated. AZ, USA. Available: http://www.factor2.com/product p/a-341.htm 
${ }^{57}$ Dow Corning Silicones. MI, USA. Available:

https://www.xiameter.com/en/Products/Pages/ProductDetail.aspx?pid=01013084\&R=X68EN\&C=US

${ }^{58}$ Factor II, Incorporated. AZ, USA. Available: http://www.factor2.com/product_p/lsr-05.htm

${ }^{59}$ Wacker Chemie. AG, Germany. Available: http://www.wacker.com/cms/en/productsmarkets/products/product.jsp?product $=12920$

${ }^{60}$ A. Almagableh, P. R. Mantena, A. Alostaz, W. Liu and L. T. Drzal, "Effects of bromination on the viscoelastic response of vinyl ester nanocomposites," J. Express Polymer Letters 3 (11), 724-732

555 (2009).

${ }^{61}$ TA Instruments, Q Series ${ }^{\mathrm{TM}}$, “Getting Started Guide,” Revision F (New Castle, Delaware, 2004).

${ }^{62}$ R. G. Brown, C. E. Hann, J. G. Chase, and L. A. Ray, "Surface reconstruction for a DIET breast cancer screening system," presented at the 17th International Federation of Automatic Conrol World Congress (IFAC), Seoul, Korea, 2008.

$560{ }^{63}$ R. G. Brown, "Three-dimensional motion capture for the DIET breast cancer imaging system," $\mathrm{PhD}$ Thesis, Department of Mechanical Engineering, University of Canterbury, 2008.

${ }^{64}$ J. G. Chase, C. E. Hann, and L. A. Ray, "Global motion invariant signature for fast and accurate motion tracking in a digital image based elasto-tomography system," United States Patent, US 8249691 B2, 21 Aug 2012.

$565{ }^{65}$ E. E. Van Houten, M. I. Miga, J. B. Weaver, F. E. Kennedy, K. D. Paulsen, "Three-dimensional subzone-based reconstruction algorithm for MR elastography," Magn. Reson. Med. 45 (5), 827-837 (2001). 